\title{
Low dose of hydroxychloroquine reduces fatality of critically ill patients with COVID-19
}

\author{
Bo $\mathrm{Yu}^{1}$, Chenze $\mathrm{Li}^{1}$, Peng Chen ${ }^{1}$, Ning Zhou ${ }^{1}$, Luyun Wang ${ }^{1}, \mathrm{Jia} \mathrm{Li}^{2}$, Hualiang Jiang ${ }^{2,3}$ \& \\ Dao-Wen Wang ${ }^{1 *}$
}

\begin{abstract}
${ }^{1}$ Division of Cardiology, Department of Internal Medicine and Hubei Key Laboratory of Genetics and Molecular Mechanisms of Cardiological Disorders, Tongji Hospital, Tongji Medical College, Huazhong University of Science and Technology, Wuhan 430030, China;

${ }^{2}$ State Key Laboratory of Drug Research, Shanghai Institute of Materia Medica, Chinese Academy of Sciences, Shanghai 201203, China;

${ }^{3}$ Shanghai Institute for Advanced Immunochemical Studies and School of Life Science and Technology, Shanghai Tech University, Shanghai 201210, China
\end{abstract}

Received April 23, 2020; accepted May 12, 2020; published online May 15, 2020

\begin{abstract}
Coronavirus disease 2019 (COVID-19) is a pandemic with no specific drugs and high fatality. The most urgent need is to find effective treatments. We sought to determine whether hydroxychloroquine (HCQ) application may reduce the death risk of critically ill COVID-19 patients. In this retrospective study, we included 550 critically ill COVID-19 patients who need mechanical ventilation in Tongji Hospital, Wuhan, from February 1, 2020 to April 4, 2020. All 550 patients received comparable basic treatments including antiviral drugs and antibiotics, and 48 of them were treated with oral HCQ treatment (200 mg twice a day for 7-10 days) in addition to the basic treatments. Primary endpoint is fatality of patients, and inflammatory cytokine levels were compared between HCQ and non-hydroxychloroquine (NHCQ) treatments. We found that fatalities are $18.8 \%(9 / 48)$ in HCQ group, which is significantly lower than $47.4 \%(238 / 502)$ in the NHCQ group $(P<0.001)$. The time of hospital stay before patient death is $15(10-21)$ days and 8 (4-14) days for the HCQ and NHCQ groups, respectively $(P<0.05)$. The levels of inflammatory cytokine IL-6 were significantly reduced from $22.2(8.3-118.9) \mathrm{pg} \mathrm{mL}^{-1}$ at the beginning of the treatment to 5.2 (3.0-23.4) $\mathrm{pg} \mathrm{mL}^{-1}(P<0.05)$ at the end of the treatment in the HCQ group but there is no change in the NHCQ group. These data demonstrate that addition of HCQ on top of the basic treatments is highly effective in reducing the fatality of critically ill patients of COVID-19 through attenuation of inflammatory cytokine storm. Therefore, HCQ should be prescribed as a part of treatment for critically ill COVID-19 patients, with possible outcome of saving lives.
\end{abstract}

hydroxychloroquine, IL-6, mortalities, COVID-19

Citation: Yu, B., Li, C., Chen, P., Zhou, N., Wang, L., Li, J., Jiang, H., and Wang, D.W. (2020). Low dose of hydroxychloroquine reduces fatality of critically ill patients with COVID-19. Sci China Life Sci 63, 1515-1521. https://doi.org/10.1007/s11427-020-1732-2

\section{INTRODUCTION}

Since the outbreak of infection by a novel severe acute respiratory syndrome coronavirus 2 (SARS-CoV-2) at the end of 2019 in Wuhan, China (Guan et al., 2020), the coronavirus disease 2019 (COVID-19) has become a worldwide pandemic, with more than $1,918,432$ infections and 123,460

\footnotetext{
*Corresponding author (email: dwwang@tjh.tjmu.edu.cn)
}

deaths as reported on April 14 of 2020, which have increased rapidly to more than 4,000,000 infections and 277,088 deaths on May 9 of 2020.

To combat this awful epidemic and help frontline physicians to treat patients and save lives, the World Health Organization (WHO), China, and many other countries have issued preliminary guidance on screening and diagnosis of infections in populations as well as managements of infection control. However, because of the lack of effective re- 
medy for COVID-19, the recommended treatment for acutely ill patients are severely limited, with options for compassionate managements that include the use of Chinese herbs (National Health Commission of China, 2020). Although these strategies help many patients, especially in China, severe and especially critically ill patients are with a high risk of death. Recent clinical trials on antiviral drugs such as Arbidol have not shown significant therapeutic benefit and the effect of Remdesivir still needs more evidence (Grein et al., 2020). Recent clinical observation suggests that Tocilizumab against interleukin 6 (IL-6), a major proinflammatory cytokine, to calm down the cytokine storm caused by SARS-CoV-2 infection, could lead to therapeutic effects in a portion of patients (Zhang et al., 2020). More recently, plasma of convalescent patients was used in Wuhan to manage refractory patients successfully, but the scope of this treatment is greatly constrained due to the limited supply of effective plasma.

Chloroquine (CQ) and hydroxychloroquine (HCQ) are two classic drugs (Rainsford et al., 2015), which were originally used to treat malaria. In recent years, repurpose of existing drugs such as chloroquine class of drugs for other diseases has received increasing attention (Rainsford et al., 2015; Plantone and Koudriavtseva, 2018). CQ and HCQ have been used to treat rheumatological and immunological diseases because of their inhibition of immunity and proinflammatory cytokines. CQ and HCQ also have antimicrobial effects and were used to treat infections by bacteria and viruses. Recently, HCQ was reported to be effective in inhibiting SARSCoV-2 infection in cell-based assays (Bian et al., 2020), and treatment with HCQ was significantly associated with reduction and disappearance of viral load in COVID-19 patients in a small-sized sample (Gautret et al., 2020), which instigated new hope for an effective treatment for COVID19.

The key to reduce fatality of COVID-19, however, is to cure critically ill patients. This study demonstrates that HCQ, a less toxic derivative of CQ, has dramatically decreased the fatality of critically ill patients of COVID-19 by attenuating the inflammatory cytokine storm.

\section{RESULTS}

\section{Study patients}

From February 1, 2020 to April 4, 2020, a total of 550 critically ill COVID-19 patients were admitted to Tongji Hospital, including 344 males and 206 females. The median age was 68 years old. Forty-eight patients received HCQ treatments (Shanghai Pharmaceutical CO., LTD, $200 \mathrm{mg} /$ tablet) (oral $200 \mathrm{mg}$ twice per day for 7-10 days), and the remaining 502 received basic treatments (non-hydroxychloroquine treatments, NHCQ). There were no difference in baseline characteristics including age, gender, original comorbidities, as well as severity of disease between these two groups (Table 1).

\section{Study outcomes}

In a total of 550 patients, 247 patients died (the fatality was $44.9 \%$ ). Nine out of 48 HCQ-treated patients died (18.8\%), while 238/502 patients from the NHCQ group died (47.4\%, $P<0.001)$. Furthermore, the average hospital stay time was 32 days in the HCQ group and 30 days in the NHCQ-treated patients $(P=0.314)$, but the hospital stay time before death from admission was longer for the HCQ patients than that for the NHCQ patients ( 15 vs. 8 days, $P=0.027$ ), suggesting that the application of HCQ prolonged the survival time of the patients (Table 2, Figure 1A).

For the in-hospital fatality, the HCQ treatment groups did not violate the proportional hazard assumption $(P=0.061)$. Thus, the proportional Cox regression was used to analyze the influence of other factors. The results showed that the use of HCQ was associated with a significantly decreased fatality risk (unadjusted HR: $0.31 ; 95 \%$ CI: $0.16-0.61$; $P=0.001$; adjusted HR: 0.36 ; $95 \%$ CI: $0.18-0.75 ; P=0.006$ ) (Table 3), which further supports that the critically ill patients can benefit substantially from HCQ treatment.

\section{Effect of HCQ on inflammatory cytokines}

Laboratory tests showed that HCQ significantly reduced IL6 levels in plasma $22.2(8.3-118.9) \mathrm{pg} \mathrm{mL}^{-1}$ vs. $5.2(3.0-$ 23.4) $\mathrm{pg} \mathrm{mL}^{-1}(P=0.002)$, but there is little change in NHCQ patients $\left(21.3(8.8-62.8) \mathrm{pg} \mathrm{mL}^{-1}\right.$ vs. 20.2 (6.1-94.4)

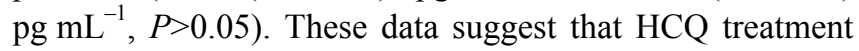
greatly lowered the inflammatory levels in critically ill COVID-19 patients (Figure 2). Furthermore, we analyzed the change of IL-6 levels during the hospitalization period and found that oral HCQ treatment rapidly resulted in reducing IL-6 level and kept the lower level stable during therapy period. However, after HCQ treatment stopped, the IL-6 level went up to control level (Figure 3).

Furthermore, we analyzed the effects of different IL-6 levels on death risk in response to HCQ treatment and found that when the IL-6 levels were higher than $60 \mathrm{pg} \mathrm{mL}^{-1}$, patients were more severe. For patients with IL-6 levels higher than $60 \mathrm{pg} \mathrm{mL}^{-1}$, HCQ treatment decreased IL-6 level from $100.0(62.6-189.4) \mathrm{pg} \mathrm{mL}^{-1}$ to $28.4(10.9-59.3) \mathrm{pg} \mathrm{mL}^{-1}$ after 5 days' treatment, and reached $9.8(5.5-24.6) \mathrm{pg} \mathrm{mL}^{-1}$ after 10 days' treatment. In contrast, in the NHCQ-treated patients, IL-6 levels were elevated from 87.5 (72.9-240.3) pg mL ${ }^{-1}$ to $92.4(34.1-199.3) \mathrm{pg} \mathrm{mL}^{-1}$ after 5 days, and reached 110.4 (31.6-235.6) $\mathrm{pg} \mathrm{mL}^{-1}$ after 10 days. Moreover, for patients with IL-6 levels higher than $60 \mathrm{pg} \mathrm{mL}^{-1}$, HCQ treatment reduced the fatality from $71.2 \%(37 / 52)$ to 
Table 1 Baseline characteristics of critically ill COVID-19 patients ${ }^{\text {a) }}$

\begin{tabular}{|c|c|c|c|c|}
\hline & All patients $(n=550)$ & HCQ $(n=48)$ & NHCQ $(n=502)$ & $P$ \\
\hline Age, years & $68(59-77)$ & $68(60-75)$ & $68(59-77)$ & 0.619 \\
\hline \multicolumn{5}{|l|}{ Age range, years } \\
\hline$\leq 60(\%)$ & $139(25.3)$ & $11(22.9)$ & $128(25.5)$ & 0.694 \\
\hline$>60(\%)$ & $411(74.7)$ & $37(77.1)$ & $374(71.9)$ & 0.694 \\
\hline Gender, male (\%) & $344(62.5)$ & $32(66.7)$ & $312(62.2)$ & 0.537 \\
\hline \multicolumn{5}{|l|}{ Original comorbidities } \\
\hline Hypertension (\%) & $252(45.8)$ & $23(47.9)$ & $229(45.6)$ & 0.760 \\
\hline Coronary heart disease (\%) & $59(10.7)$ & $2(4.2)$ & $57(11.4)$ & 0.147 \\
\hline COPD (\%) & $16(2.9)$ & $0(0)$ & $16(3.2)$ & 0.383 \\
\hline Diabetes $(\%)$ & $94(17.1)$ & $12(25.0)$ & $82(16.3)$ & 0.128 \\
\hline \multicolumn{5}{|l|}{ Vital signs } \\
\hline Body temperature, ${ }^{\circ} \mathrm{C}$ & $36.6(36.3-37.0)$ & $36.7(36.2-37.3)$ & $36.7(36.3-37.3)$ & 0.704 \\
\hline Pulse, beats $\min ^{-1}$ & $89(80-102)$ & $91(80-103)$ & $92(81-107)$ & 0.594 \\
\hline Respiratory rate, breaths $\min ^{-1}$ & $20(20-22)$ & $25(20-30)$ & $21(20-26)$ & 0.052 \\
\hline Systolic blood pressure, $\mathrm{mmHg}$ & $130(119-144)$ & $131(117-149)$ & $133(118-148)$ & 0.789 \\
\hline Diastolic blood pressure, $\mathrm{mmHg}$ & $80(72-89)$ & $79(70-90)$ & $80(71-88)$ & 0.608 \\
\hline $\mathrm{SpO}_{2}$ on admission $(\%)$ & $97(95-98)$ & $95(90-96)$ & $96(88-98)$ & 0.216 \\
\hline \multicolumn{5}{|l|}{ Symptoms, number/total number (\%) } \\
\hline Fever & $354 / 458(77.3)$ & $29 / 43(67.4)$ & $325 / 415(78.3)$ & 0.105 \\
\hline Cough & $312 / 458(68.1)$ & $29 / 43(67.4)$ & $283 / 415(68.2)$ & 0.920 \\
\hline Sputum production & $223 / 458(48.7)$ & $26 / 43(60.5)$ & $197 / 415(47.5)$ & 0.105 \\
\hline Chest tightness & $65 / 458(14.2)$ & $7 / 43(16.3)$ & $58 / 415(14.0)$ & 0.680 \\
\hline Shortness of breath & $221 / 458(48.3)$ & $30 / 43(69.8)$ & $191 / 415(46.0)$ & 0.003 \\
\hline Nasal congestion & $4 / 458(0.9)$ & $0 / 43(0)$ & $4 / 415(1.0)$ & 1 \\
\hline Nausea & $18 / 458(3.9)$ & $1 / 43(2.3)$ & $17 / 415(4.1)$ & 1 \\
\hline Diarrhea & $100 / 458(21.8)$ & 9/43 (20.9) & $91 / 415$ (21.9) & 0.880 \\
\hline Muscle aches & $32 / 458(0.7)$ & $1 / 43(2.3)$ & $31 / 415(7.5)$ & 0.344 \\
\hline Pharynx discomfort & $16 / 458(3.5)$ & $2 / 43(4.7)$ & $14 / 415(3.4)$ & 0.655 \\
\hline Fatigue & $87 / 458(19.0)$ & $8 / 43(18.6)$ & $79 / 415(19.0)$ & 0.945 \\
\hline \multicolumn{5}{|l|}{ Laboratory parameters, } \\
\hline White-cell count, $\times 10^{9} \mathrm{~L}^{-1}$ & $7.7(5.5-11.4)$ & $7.3(5.3-12.1)$ & $7.7(5.5-11.4)$ & 0.923 \\
\hline Lymphocyte count, $\times 10^{9} \mathrm{~L}^{-1}$ & $0.7(0.5-1.0)$ & $0.7(0.5-1.0)$ & $0.7(0.5-1.0)$ & 0.923 \\
\hline Neutrophil count, $\times 10^{9} \mathrm{~L}^{-1}$ & $6.2(4.2-10.1)$ & $6.3(4.2-9.5)$ & $6.2(4.2-10.2)$ & 0.909 \\
\hline Platelet count, $\times 10^{9} \mathrm{~L}^{-1}$ & $182.0(129.8-255.0)$ & $189.0(137.0-257.0)$ & $182.0(128.5-255.0)$ & 0.879 \\
\hline Hemoglobin, $\mathrm{g} \mathrm{L}^{-1}$ & $125.0(109.0-139.0)$ & $121.0(107.5-134.5)$ & $125.0(110.0-139.0)$ & 0.424 \\
\hline Alanine aminotransferase, $\mathrm{U} \mathrm{L}^{-1}$ & $26.0(16.0-41.0)$ & $30.0(22.0-52.0)$ & $25.0(16.0-40.0)$ & 0.009 \\
\hline Aspartate aminotransferase, $\mathrm{U} \mathrm{L}^{-1}$ & $35.0(23.0-53.0)$ & $42.0(20.0-60.0)$ & $34.0(24.0-52.0)$ & 0.468 \\
\hline Total bilirubin, $\mu \mathrm{mol} \mathrm{L} \mathrm{L}^{-1}$ & $10.8(7.8-15.7)$ & $11.0(8.2-15.5)$ & $10.7(7.7-15.8)$ & 0.938 \\
\hline Albumin, $\mathrm{g} \mathrm{L}^{-1}$ & $31.4(28.7-34.5)$ & $31.1(29.6-33.6)$ & $31.5(28.7-34.6)$ & 0.527 \\
\hline Lactate dehydrogenase, $\mathrm{U} \mathrm{L}^{-1}$ & $395.5(293.0-536.0)$ & $401.0(311.5-493.0)$ & $395.0(291.5-541.0)$ & 0.949 \\
\hline Creatinine, $\mu \mathrm{mol} \mathrm{L}{ }^{-1}$ & $81.0(62.0-103.0)$ & $86.0(63.0-110.5)$ & $79.0(62.0-103.0)$ & 0.440 \\
\hline Blood urea nitrogen, $\mathrm{mmol} \mathrm{L}^{-1}$ & $6.4(4.4-10.4)$ & $5.9(4.1-9.6)$ & $6.5(4.5-10.7)$ & 0.392 \\
\hline International normalized ratio & $1.1(1.1-1.3)$ & $1.1(1.1-1.2)$ & $1.2(1.1-1.3)$ & 0.034 \\
\hline D-dimer, $\mathrm{mg} \mathrm{L}^{-1}$ & $2.3(1.1-9.6)$ & $2.6(1.0-10.1)$ & $2.3(1.1-9.7)$ & 0.895 \\
\hline APTT, s & $40.3(36.6-45.4)$ & $39.0(36.2-50.7)$ & $40.4(36.6-44.9)$ & 0.962 \\
\hline C-reactive protein, $\mathrm{mg} \mathrm{L}^{-1}$ & $75.7(36.3-128.9)$ & $84.1(27.5-117.4)$ & $75.6(37.1-131.5)$ & 0.789 \\
\hline NT-ProBNP, pg mL ${ }^{-1}$ & $598.5(223.5-1814.3)$ & $600.5(191.8-1926.5)$ & $598.5(224.5-1829.8)$ & 0.803 \\
\hline cTnI, pg mL $\mathrm{m}^{-1}$ & $15.9(6.2-59.5)$ & $12.1(6.5-47.2)$ & $16.3(6.2-60.7)$ & 0.582 \\
\hline IL-6, pg mL ${ }^{-1}$ & $37.4(14.0-96.1)$ & $25.3(12.0-111.1)$ & $31.6(14.0-95.9)$ & 0.593 \\
\hline IL-10, $\mathrm{gg} \mathrm{mL}^{-1}$ & $6.5(5.0-12.7)$ & $6.8(5.0-9.3)$ & $6.5(5.0-13.5)$ & 0.706 \\
\hline IL-8, pg mL ${ }^{-1}$ & $22.4(12.0-43.8)$ & $23.1(10.4-30.8)$ & $22.2(12.1-47.5)$ & 0.471 \\
\hline TNF- $\alpha, \mathrm{pg} \mathrm{mL}^{-1}$ & $10.6(8.2-14.6)$ & $11.0(9.1-13.7)$ & $10.4(8.2-14.8)$ & 0.596 \\
\hline IL- $1 \beta$, pg mL ${ }^{-1}$ & $5.0(5.0-5.0)$ & $5.0(5.0-5.3)$ & $5.0(5.0-5.0)$ & 0.524 \\
\hline IL-2R, $\mathrm{U} \mathrm{mL}^{-1}$ & $1026.0(679.0-1501.0)$ & $977.5(749.0-1544.8)$ & $1026.0(672.0-1490.5)$ & 0.592 \\
\hline Oxygen therapy, number (\%) & $545(99.1)$ & 47 (97.9) & $498(99.2)$ & 0.368 \\
\hline Mechanical ventilation, number (\%) & $349(63.5)$ & $28(58.3)$ & $321(63.9)$ & 0.441 \\
\hline
\end{tabular}

a) Mechanical ventilation contained non-invasive ventilation and invasive ventilation. Data are presented as medians and interquartile range (Q1-Q3). HCQ, hydroxychloroquine treatment; NHCQ, non-hydroxychloroquine treatment; COPD, chronic obstructive pulmonary disease; APTT, activated partial thromboplastin time; NT-proBNP, N-terminal pro-B-type natriuretic peptide; cTnI, cardiac troponin I; IL, interleukin; TNF- $\alpha$, tumor necrosis factor- $\alpha$; SpO2, percutaneous oxygen saturation. 


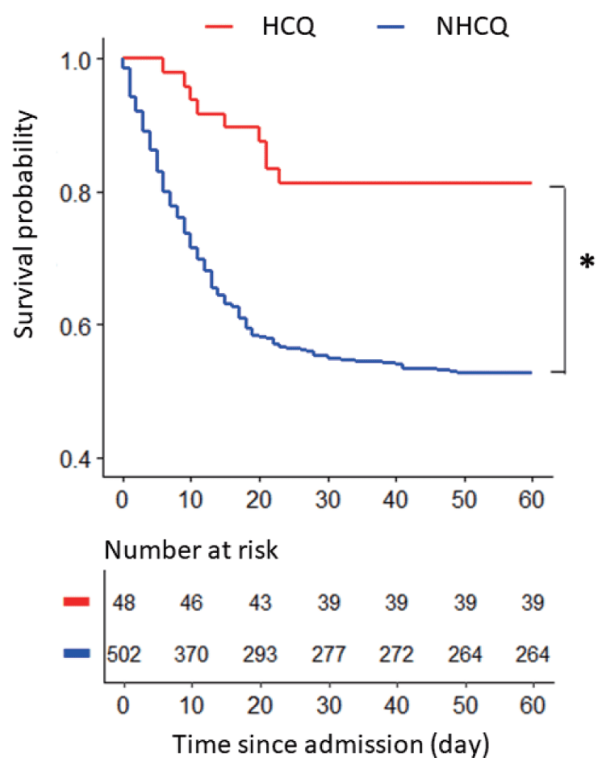

Figure 1 Kaplan-Meier curve of COVID-19 patients treated with or without HCQ. HCQ treatment significantly reduced the fatality of critically ill COVID-19 patients compared with NHCQ treated patients $\left({ }^{*} P<0.001\right)$.

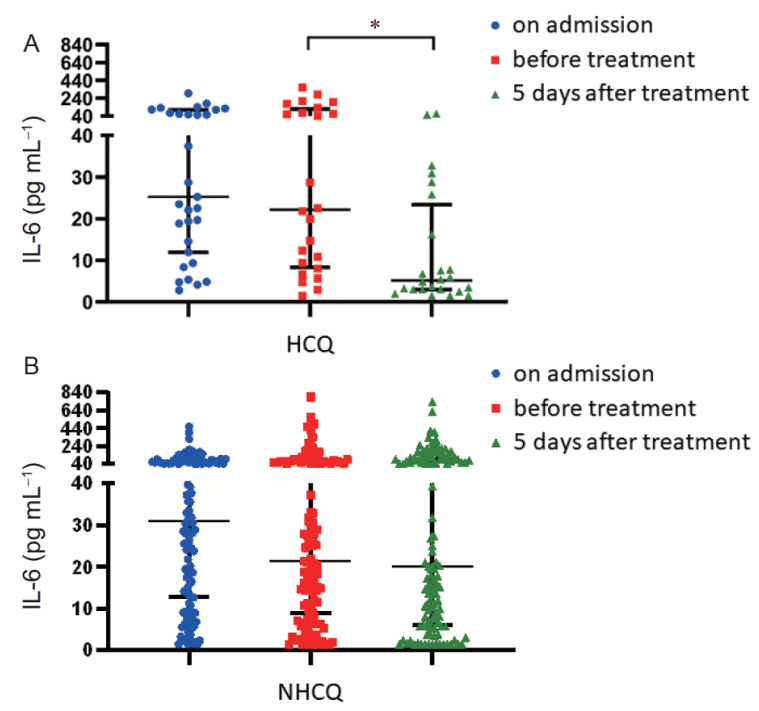

Figure 2 Effects of HCQ treatment on plasma levels of IL-6. HCQ treatment significantly decreased the levels of IL-6 in about 5 (4-8) days, $* P<0.05$ (A). The levels of IL-6 were not significantly decreased in NHCQ treatment group at the same observation time (B). Data are presented as medians and interquartile range (Q1-Q3).

44.4\% (4/9) in the control $(P>0.05)$. For patients with IL-6 levels lower than $60 \mathrm{pg} \mathrm{mL}^{-1}$ before treated with HCQ, HCQ decreased IL-6 levels from $12.8(8.2-23.5) \mathrm{pg} \mathrm{mL}^{-1}$ to 3.5

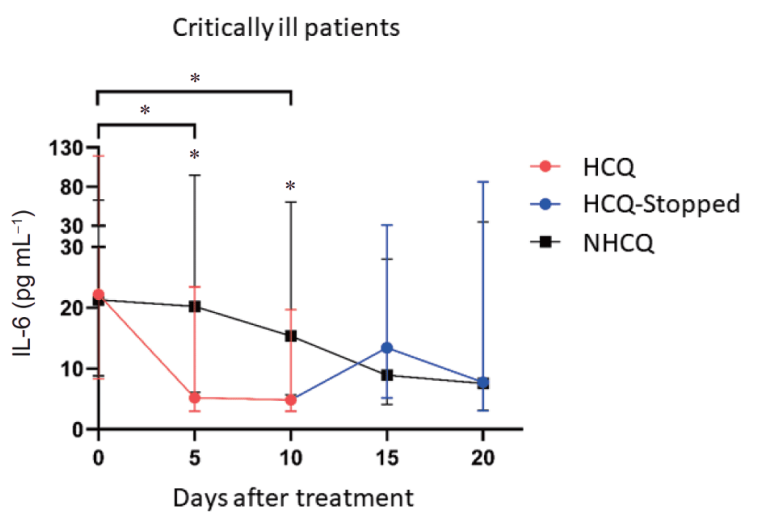

Figure 3 Continuous curves of plasma levels of IL-6 showing that the consistency of HCQ treatment period with lowering IL-6 levels, ${ }^{*} P<0.05$. Data are presented as medians and interquartile range (Q1-Q3).

(2.7-5.6) $\mathrm{pg} \mathrm{mL} \mathrm{mL}^{-1}$ after 5 days, and reached 3.0 (2.1-14.4) pg mL ${ }^{-1}$ after 10 days. In the NHCQ patients, IL-6 levels decreased only slightly from $15.0(6.4-26.2) \mathrm{pg} \mathrm{mL}^{-1}$ to 13.8 (5.7-43.1) $\mathrm{pg} \mathrm{mL}^{-1}$ after 5 days, and reached $10.8(5.1-50.0)$ $\mathrm{pg} \mathrm{mL}{ }^{-1}$ after 10 days. The fatality was $6.3 \%(1 / 16)$ in HCQ patients and $21.3 \%(27 / 127)$ in NHCQ group $(P>0.05)$ for these patients with initial IL-6 levels lower than $60 \mathrm{pg} \mathrm{mL} \mathrm{m}^{-1}$. These results indicated that the patients with higher IL-6 levels were severer and had higher fatality than those with lower IL-6 levels, and HCQ had stronger effect on reducing IL-6 levels in these severer patients.

We also analyzed the effect of HCQ treatment starting time after admission on curative response and found that the median time to start HCQ treatment was 10 (3-13) days after admission. Interestingly, the early start of HCQ treatment (within 5 days after admission) resulted in lower fatality than late start of HCQ treatment (5 days after admission, 1/11 $(9.1 \%)$ vs. $8 / 37(21.6 \%))$, which suggests that HCQ treatment should be started earlier after admission, although the result is not statistically significant $(P>0.05)$.

\section{DISCUSSION}

This retrospective study found that HCQ treatment was associated significantly with the reduced fatality of critically ill patients with COVID-19 and greatly lowered the levels of IL-6, one of the most inflammatory cytokines. We also found that the time period that led to death in the HCQ-treated

Table 2 Comparison of clinical outcomes between HCQ-treated and NHCQ-treated patients ${ }^{\text {a) }}$

\begin{tabular}{|c|c|c|c|}
\hline & HCQ & NHCQ & $P$ \\
\hline Total patients, $n$ & 48 & 502 & \\
\hline Dead patients, $n(\%)$ & $9(18.8)$ & $238(47.4)$ & $<0.001$ \\
\hline Hospital stay time before death (d) & $15(10-21)$ & $8(4-14)$ & 0.027 \\
\hline
\end{tabular}

a) Data are presented as medians and interquartile range (Q1-Q3). HCQ, hydroxychloroquine treatment; NHCQ, non-hydroxychloroquine treatment. 
Table 3 Univariable and multivariable cox proportional hazards model for 60-day fatality after HCQ treatment ${ }^{\text {a) }}$

\begin{tabular}{|c|c|c|}
\hline & Crude HR $(95 \% \mathrm{CI}), P$ & Adjusted HR $(95 \% \mathrm{CI}), P^{*}$ \\
\hline HCQ-treated & $0.31(0.16-0.61), 0.001$ & $0.36(0.18-0.75), 0.006$ \\
\hline Age & $1.02(1.01-1.03),<0.001$ & $1.02(1.01-1.03), 0.004$ \\
\hline Gender & 1.45 (1.11-1.9), 0.006 & $1.64(1.18-2.29), 0.003$ \\
\hline Hypertension & $0.73(0.57-0.94), 0.017$ & $0.77(0.56-1.05), 0.093$ \\
\hline Coronary heart disease & $1.21(0.84-1.76), 0.313$ & $1.11(0.71-1.72), 0.656$ \\
\hline COPD & $1.12(0.55-2.26), 0.761$ & $0.77(0.31-1.93), 0.583$ \\
\hline Diabetes & $0.64(0.45-0.93), 0.018$ & $0.57(0.35-0.92), 0.023$ \\
\hline Body temperature & $1.34(1.16-1.56),<0.001$ & $1.3(1.09-1.54), 0.003$ \\
\hline Pulse & $1(1-1), 0.526$ & - \\
\hline Respiratory rate & $1(1-1.01), 0.349$ & - \\
\hline Systolic blood pressure & $1(0.99-1), 0.401$ & - \\
\hline Diastolic blood pressure & $1(0.99-1), 0.104$ & - \\
\hline $\mathrm{SpO}_{2}$ on admission & $0.96(0.95-0.97),<0.001$ & $0.97(0.96-0.99),<0.001$ \\
\hline Fever & $0.87(0.63-1.19), 0.384$ & - \\
\hline Cough & $0.79(0.6-1.06), 0.118$ & - \\
\hline Shortness of breath & $1.05(0.8-1.39), 0.709$ & - \\
\hline Oxygen therapy & $0.93(0.23-3.73), 0.915$ & $0.14(0.03-0.63), 0.01$ \\
\hline Mechanical ventilation & $7.4(4.88-11.2),<0.001$ & $8.07(4.83-13.47),<0.001$ \\
\hline
\end{tabular}

a) ${ }^{*}$, The variables with a $P$ value less than 0.01 in the univariable Cox models, and those representing the history of diseases were included into the multivariable analysis. HCQ, hydroxychloroquine treatment.

group is longer, suggesting that HCQ prolonged the survival time of critically ill patients even if their lives were eventually lost, which further supports that HCQ has therapeutic effects for critically ill patients with COVID-19. Importantly, we found that the role of HCQ in lowering plasma IL-6 levels was highly consistent with the duration of its administration, and once the drug was stopped, plasma IL-6 levels returned to the control levels.

We have noticed that HCQ has in vitro antiviral effects on several viruses, including SARS and SARS-CoV-2 (Al-Bari, 2015; Bian et al., 2020; Gautret et al., 2020; Yao et al., 2020), which may contribute directly to its therapeutic effect on COVID-19 patients. However, the anti-SARS-CoV2 action of HCQ requires a much higher concentration of HCQ than the dose used in this study, and cannot account for the significant efficiency on critically ill patients in our cohort.

COVID-19 patients displayed apparent immunity disorders, showing marked reduction in lymphocyte numbers in peripheral blood and lymphatic tissues, but large amounts of lymphocyte infiltration in lungs as well as in other critical organs such as the heart. Many patients showed symptoms of cytokine storm with markedly elevated levels of the proinflammatory cytokine IL-6, suggesting over-activated immune responses (Bian et al., 2020). Anti-IL-6 antibody, Tocilizumab, has been shown to be an effective treatment option for COVID-19 patients with a risk of cytokine storms (Luo et al., 2020). In this study, we demonstrate that HCQ can mimic the effect of anti-IL-6 antibody by greatly redu- cing the levels of Il-6 in the critically ill COVID-19 patients. In addition, HCQ can modulate human inflammatory macrophage polarization via downregulating M1 but upregulating M2 macrophages (Shiratori et al., 2018; Sarzi-Puttini et al., 2020), and inhibit proinflammatory cytokines through inhibition of lysosomal-autophagy pathways (He et al., 2011) and formation of double membrane vesicles (He et al., 2011; Plantone and Koudriavtseva, 2018), a process required for genome replication by the SARS coronavirus replication complex (Snijder et al., 2006). Thus, the anti-inflammatory action of HCQ in combination with its inhibitory activity of viral replication may contribute greatly to its therapeutic effects on critically ill COVID-19 patients. In addition, tissue distribution of HCQ is unique and favors therapy of pneumonia because it has much higher concentrations in the lungs (McChesney et al., 1967). Importantly, the CQ class of drugs has been shown to repress inflammation with great synergy with low concentrations of glucocorticoids, one of the most potent classes of anti-inflammatory drugs, pointing to the possibility of combined therapy of HCQ with a low dose of glucocorticoids (He et al., 2011).

Additionally, lower plasma levels of IL-6 after HCQ treatment are highly correlated with the application period of HCQ. IL-6 levels returned quickly to the levels of NHCQ patients after HCQ treatment was stopped. This finding indicates that we need to adjust our treatment regimen by extending the use of HCQ until patients are completely cured.

We also noticed a couple of negative reports about CQ and 
HCQ (Geleris et al., 2020; Mahevas et al., 2020; Magagnoli et al., 2020). However, based on the results in these studies, it is very likely that they used high doses of HCQ, which might induce cardiotoxicity and death. They might also have used high doses of HCQ as antiviral agents rather than for antiinflammation. In contrast, the dose of HCQ used in the present study is much lower than that in previous studies but is the same as used for treating inflammatory diseases, and thus HCQ for COVID-19 patients works more likely as an agent for anti-inflammation and immunomodulation, rather than as a direct antiviral agent. Recently, Geleris et al. (2020) reported their observation study, showing that there was no significant association between hydroxychloroquine use and intubation or death. By carefully re-analyzing this report, however, we noticed that in this study, HCQ-treated patients were more severely ill at baseline (even if after propensity score-matched) than NHCQ-treated patients. In addition, the time of treatment for patients is too short (5 days) to show the efficacy of HCQ. Another concern of this study is that about 60\% HCQ-treated patients received azithromycin simultaneously, which increases risk of QT-interval prolongation and sudden death.

In summary, this retrospective study demonstrates that HCQ significantly reduces death risk of critically ill COVID19 patients without apparent toxicity, and its mechanism of action is likely mediated through its inhibition of inflammatory cytokines on top of its ability in inhibiting viral replication. Therefore, despite the need for randomizeddouble blind control study, HCQ should be considered as an additional option to treat critically ill COVID-19 patients, which could save lives during the current COVID-19 pandemic. Although this retrospective study was performed on critically ill COVID-19 patients, HCQ could also be an option for patients at an early stage considering its safety records and the long history of its use in treating malaria infections. This study has some limitations. For example, it is a retrospective study and HCQ treatment group only has 48 cases, much less than the number in NHCQ group. Therefore, large-scale random clinical studies are necessary for the treatment of COVID-19 by HCQ.

\section{METHODS}

\section{Study design}

This investigation is a retrospective study involving critically ill patients with COVID-19 in Tongji Hospital, Wuhan, China. These patients were diagnosed according to the WHO interim guideline and the Clinical Guideline for COVID-19 Diagnosis and Treatment published by the National Health Commission of China between February 1, 2020 and April 4, 2020. This study has been approved by the institutional review board of Tongji Hospital (IRBID: TJ-C20200113). The written informed consent has been waived by the Ethics Committee because of the retrospective and anonymous nature of the data.

\section{Patients' information}

All patients have medical history and imaging characteristics of COVID-19 and have been confirmed with SARS-CoV-2 infection by laboratory tests. In addition, all patients were confirmed by chest computed tomography (CT) and SARS$\mathrm{CoV}-2$ pathogenic test. The inclusion as critically ill patients had to meet one of the following criteria: (i) patients had respiratory failure and needed mechanical ventilation; (ii) patients had septic shock during hospitalization; (iii) patients with other organ failures that required monitoring and treatment by intensive care unit. In this study, we included all critically ill adult patients (age $>18$ years old) with hospitalization during the epidemic period from February 1, 2020 to April 4, 2020.

The baseline treatments were comparable for these two groups, including application of antiviral drugs (Lopinavir and Ritonavir, Entecavir hydrate, or Ribavirin) with $41.7 \%$ and $42.0 \%$ patients in HCQ and NHCQ, respectively $(P=0.96)$; intravenous immunoglobulin in $52.1 \%$ patients in HCQ and $48.6 \%$ patients in NHCQ, respectively $(P=0.65)$; immunoenhancer in $16.7 \%$ patients in HCQ and $17.7 \%$ patients in NHCQ, respectively $(P=0.86)$; antibiotics in $77.1 \%$ patients in HCQ and $87.1 \%$ patients in NHCQ, respectively $(P=0.06)$, but interferon application in $0 \%$ patients in HCQ and $10.8 \%$ patients in NCHQ $(P=0.01)$.

\section{Data collection of patients}

Health care data of COVID-19 patients, including medical history, chest CT, and laboratory tests, in-hospital therapies, and clinical deposits (death or cured discharge), were extracted by data coordinators through the electronic medical records. Laboratory test results included inflammatory cytokines and counts of white blood cells in peripheral blood.

\section{Statistical analysis}

Continuous values were expressed as medians and interquartile range (Q1-Q3) and categorical variables as counts and percentages. The comparisons of continuous values between groups were performed with Wilcoxon rank-sum tests. For paired continuous variables, paired sample Wilcoxon rank test was used. Categorical variables were compared using Chi-square test. Survival curves were described by Kaplan-Meier method and compared with the log-rank test. For the test of primary null hypothesis, the hazard ratios and $95 \%$ confidence interval were calculated by proportional Cox regression models if Schoenfeld individual test did not 
show a violation of proportional-hazards assumption and extended Cox model where treatment groups were included as a time-varying covariate if they did. All statistical analyses were performed with $\mathrm{R}$ packages (version 3.1.4, Vienna, Austria). $P<0.05$ was considered to indicate statistical significance, and all comparisons were two-sided.

Compliance and ethics The author(s) declare that they have no conflict of interest. No funding bodies had any role in study design, data collection and analysis, decision to publish, or preparation of the manuscript.

Acknowledgements This work was supported in part by projects from Ministry of Science and Technology of China (2020YFC0844500), the National Natural Science Foundation of China (31130031), Emergency Project Fund of Chinese Academy of Sciences (2020YJFK0105) and Chinese Academy of Engineering and Ma Yun Foundation (2020-CMKYGG-05). We thank all our colleagues from the Department of Internal Medicine, Tongji Hospital, as well as all the medical staff fighting against COVID-19, for their tremendous efforts. We also thank Professor H. Eric Xu in Shanghai Institute of Materia Medica, Chinese Academy of Sciences for his great help in writing this paper.

\section{References}

Al-Bari, M.A.A. (2015). Chloroquine analogues in drug discovery: new directions of uses, mechanisms of actions and toxic manifestations from malaria to multifarious diseases. J Antimicrob Chemother 70, 16081621.

Bian, X., Shi, Z., Chen, R., Cai, J., Wang, C., Xie, J., Zhao, L., Fei, X., Zhang, H., Tan, Y., et al. (2020). Aveolar macrophage activation and cytokine storm in the pathogenesis of severe COVID-19. Immunol Pathol, https://doi.org/10.21203/rs.3.rs-19346/v1.

Gautret, P., Lagier, J.C., Parola, P., Hoang, V.T., Meddeb, L., Mailhe, M., Doudier, B., Courjon, J., Giordanengo, V., Vieira, V.E., et al. (2020). Hydroxychloroquine and azithromycin as a treatment of COVID-19: results of an open-label non-randomized clinical trial. Int J Antimicrob Ag 20, 105949.

Geleris, J., Sun, Y., Platt, J., Zucker, J., Baldwin, M., Hripcsak, G., Labella, A., Manson, D., Kubin, C., Barr, R.G., et al. (2020). Observational Study of Hydroxychloroquine in Hospitalized Patients with Covid-19. N Engl J Med https://doi/org/10.1056/NEJMoa2012410.

Grein, J., Ohmagari, N., Shin, D., Diaz, G., Asperges, E., Castagna, A., Feldt, T., Green, G., Green, M.L., Lescure, F.X., et al. (2020). Compassionate use of remdesivir for patients with severe Covid-19. N Engl J Med https://doi.org/10.1056/NEJMoa2007016.

Guan, W.J., Ni, Z.Y., Hu, Y., Liang, W.H., Ou, C.Q., He, J.X., Liu, L., Shan, H., Lei, C.L., Hui, D.S.C., et al. (2020). Clinical characteristics of coronavirus disease 2019 in China. N Engl J Med 382, 1708-1720.

He, Y., Xu, Y., Zhang, C., Gao, X., Dykema, K.J., Martin, K.R., Ke, J.,
Hudson, E.A., Khoo, S.K., Resau, J.H., et al. (2011). Identification of a lysosomal pathway that modulates glucocorticoid signaling and the inflammatory response. Sci Signal 4, ra44.

Luo, P., Liu, Y., Qiu, L., Liu, X., Liu, D., and Li, J. (2020). Tocilizumab treatment in COVID-19: a single center experience. J Med Virol https:// doi.org/10.1002/jmv.25801.

Magagnoli, J., Narendran, S., Pereira, F., Cummings, T., Hardin, J., Sutton, S.S., and Ambati, J. (2020). Outcomes of hydroxychloroquine usage in United States veterans hospitalized with Covid-19. MedRxiv, https:// doi.org/10.1101/2020.04.16.20065920.

Mahevas, M., Thi Tran, V., Roumier, M., Chabrol, A., Paule, R., Guillaud, C., Gallien, S., Lepeule, R., Szwebel, T.A., Lescure, X., et al. (2020). No evidence of clinical efficacy of hydroxychloroquine in patients hospitalized for COVID-19 infection with oxygen requirement: results of a study using routinely collected data to emulate a target trial. MedRxiv, https://doi.org/10.1101/2020.04.10.20060699.

McChesney, E.W., Banks Jr., W.F., and Fabian, R.J. (1967). Tissue distribution of chloroquine, hydroxychloroquine, and desethylchloroquine in the rat. Toxicol Appl Pharmacol 10, 501-513.

National Health Commission of China. (2020). Chinese management guideline for COVID-19 (version 5.0). http://wwwnhcgoven/yzygj/ s7653p/202002/d4b895337e19445f8d728fcafle3e13a.shtml.

Plantone, D., and Koudriavtseva, T. (2018). Current and future use of chloroquine and hydroxychloroquine in infectious, immune, neoplastic, and neurological diseases: a mini-review. Clin Drug Invest 38, 653-671.

Rainsford, K.D., Parke, A.L., Clifford-Rashotte, M., and Kean, W.F. (2015). Therapy and pharmacological properties of hydroxychloroquine and chloroquine in treatment of systemic lupus erythematosus, rheumatoid arthritis and related diseases. Inflammopharmacology 23, 231-269.

Sarzi-Puttini, P., Giorgi, V., Sirotti, S., Marotto, D., Ardizzone, S., Rizzardini, G., Antinori, S., and Galli, M. (2020). COVID-19, cytokines and immunosuppression: what can we learn from severe acute respiratory syndrome? Clin Exp Rheumatol 38, 337-342.

Shiratori, H., Feinweber, C., Luckhardt, S., Wallner, N., Geisslinger, G., Weigert, A., and Parnham, M.J. (2018). An in vitro test system for compounds that modulate human inflammatory macrophage polarization. Eur J Pharmacol 833, 328-338.

Snijder, E.J., van der Meer, Y., Zevenhoven-Dobbe, J., Onderwater, J.J.M., van der Meulen, J., Koerten, H.K., and Mommaas, A.M. (2006). Ultrastructure and origin of membrane vesicles associated with the severe acute respiratory syndrome coronavirus replication complex. JVI $80,5927-5940$.

Yao, X., Ye, F., Zhang, M., Cui, C., Huang, B., Niu, P., Liu, X., Zhao, L., Dong, E., Song, C., et al. (2020). In vitro antiviral activity and projection of optimized dosing design of hydroxychloroquine for the treatment of severe acute respiratory syndrome coronavirus 2 (SARSCoV-2). Clin Infect Dis https://doi.org/10.1093/cid/ciaa237.

Zhang, C., Wu, Z., Li, J.W., Zhao, H., and Wang, G.Q. (2020). The cytokine release syndrome (CRS) of severe COVID-19 and Interleukin6 receptor (IL-6R) antagonist Tocilizumab may be the key to reduce the mortality. Int J Antimicrob Ag 29, 105954. 